OPEN ACCESS

Edited by:

Mechthild Pohlschroder

University of Pennsylvania, USA

Reviewed by:

Jason W. Cooley,

University of Missouri, USA

Jerry Eichler,

Ben Gurion University of the Negev,

Israel

*Correspondence:

Tessa E. F. Quax,

Molecular Biology of Archaea, Institute for Biology II - Microbiology,

University of Freiburg,

Schänzlestrasse 1, 79104 Freiburg,

Germany

tessa.quax@biologie.uni-freiburg.de

Specialty section:

This article was submitted to Microbial Physiology and Metabolism,

a section of the journal

Frontiers in Microbiology

Received: 07 January 2015

Accepted: 19 May 2015

Published: 05 June 2015

Citation:

Quemin ERJ and Quax TEF (2015) Archaeal viruses at the cell envelope:

entry and egress.

Front. Microbiol. 6:552.

doi: 10.3389/fmicb.2015.00552

\section{Archaeal viruses at the cell envelope: entry and egress}

\author{
Emmanuelle R. J. Quemin ${ }^{1}$ and Tessa E. F. Quax ${ }^{2 *}$ \\ ${ }^{1}$ Department of Microbiology, Institut Pasteur, Paris, France, ${ }^{2}$ Molecular Biology of Archaea, Institute for Biology II - \\ Microbiology, University of Freiburg, Freiburg, Germany
}

The cell envelope represents the main line of host defense that viruses encounter on their way from one cell to another. The cytoplasmic membrane in general is a physical barrier that needs to be crossed both upon viral entry and exit. Therefore, viruses from the three domains of life employ a wide range of strategies for perforation of the cell membrane, each adapted to the cell surface environment of their host. Here, we review recent insights on entry and egress mechanisms of viruses infecting archaea. Due to the unique nature of the archaeal cell envelope, these particular viruses exhibit novel and unexpected mechanisms to traverse the cellular membrane.

Keywords: archaea, archaeal virus, bacterial virus, virion entry, virion egress, archaeal membrane, pili, lysis

\section{Introduction}

Members of the three domains of life, Archaea, Bacteria and Eukarya, are all subject to viral infections. Viruses have been isolated from various environments, where they are often abundant, outnumbering prokaryotic cells by a factor of 10 (Bergh et al., 1989; Borsheim et al., 1990; Suttle, 2007). Viruses infecting archaea tend to display high morphological and genetic diversity compared to viruses of bacteria and eukaryotes (Pina et al., 2011). Several archaeal viral families have members, which display unique shapes that are not found amongst other viruses, such as a bottle, droplet or spiral (Prangishvili, 2013).

The cell envelope represents a major barrier for all viruses. In fact, the cell membrane has to be traversed twice by viruses to establish successful infection, first upon entry and secondly during exit. In order to cross the cell envelope, viruses have developed various strategies, each adapted to the membrane environment of their host.

The combination of high-throughput approaches with more classical techniques has shed light on the process of viral entry and release in some archaeal virus-host model systems. However, the detailed molecular mechanisms underlying the various stages of the viral life cycle remain poorly understood in archaea in general (Quemin et al., 2014). Recently, a few studies have focused on the adsorption at the surface of the archaeal host cell before viral entry and release of viral particles at the end of the infection cycle (Bize et al., 2009; Brumfield et al., 2009; Ceballos et al., 2012; Quemin et al., 2013; Deng et al., 2014). This has delivered the very first insights into the fashion in which viruses interact with the archaeal membrane.

The cell surface of archaea is fundamentally different from bacteria (Albers and Meyer, 2011). Archaeal membranes have an alternative lipid composition and generally lack a cell wall of peptidoglycan. In addition, the motility structures present at the surface of archaea are constructed from different building blocks than their bacterial counterparts (Pohlschroder et al., 2011). Gram positive bacteria contain a lipid bilayer covered by a thick peptidoglycan cell wall and gram negative cells are surrounded by two membranes with a thinner peptidoglycan in the periplasmic space in between. While bacteria typically contain a cell wall polymer of peptidoglycan (Typas et al., 2012), 
peptidoglycan cell walls are absent from archaea. Instead, most archaea are surrounded by a thin proteinaceous surface layer (S-layer) that consists of glycosylated proteins, which are anchored in the cell membrane. In contrast to the peptidoglycan, which has a molecular composition that can be very similar from one species to another, S-layer proteins show a great diversity (Fagan and Fairweather, 2014). Hence, archaea exhibit specific features, in particular at the cell surface, which are not shared with bacteria and influence the mechanisms at play in the course of infection.

The first studies on archaeal viral entry and egress have shown that some archaeal viruses employ entry strategies that superficially resemble those of bacterial viruses (Quemin et al., 2013; Deng et al., 2014), while others utilize surprisingly novel exit mechanisms (Brumfield et al., 2009; Quax et al., 2011). Here we will give an overview of the first studies reporting viral interaction with the archaeal cell envelope, focusing on hyperthermophilic crenarchaeal viruses. Furthermore, current research permits comparison with corresponding mechanisms taking place during the viral cycle of bacterial viruses. We will discuss how features of cell surfaces compel viruses to employ specific strategies for entry and egress.

\section{Viral Entry}

A virus is able to infect only a few strains or species. Such specificity in interaction of viruses with their host is determined by the characteristics of entry, which in turn rely on the nature and structural peculiarities of the cell envelope. Adsorption as the first key step of the viral cycle is one of the most restrictive in terms of host range, depending on the accessibility and number of receptors present at the cell surface (Poranen et al., 2002). Structural proteins are found within the viral particle in metastable conformation and it is the interaction with the host cell, which leads to a more stable, lower-energy conformation of these proteins (Dimitrov, 2004). Indeed, virus entry and genome uncoating are energydependent processes and irreversible conformational change of the capsid proteins (CP) during adsorption triggers the release of the genome from the extracellular virions (Molineux and Panja, 2013). As a general rule, entry can be subdivided in two steps. For the well-studied viruses infecting bacteria, the first contact with the host is reversible and then, viruses attach irreversibly to a specific, saturable cell envelope receptor. Primary and secondary adsorptions can take place with the same receptor or, more frequently involve different players. Common cellular determinants in bacteria are peptidoglycan, lipopolysaccharide S (LPS), or cellular appendages (Poranen et al., 2002). Subsequently, delivery of the viral genome into the cellular cytoplasm happens through the cell wall and bacterial membrane. Indeed, the nature of the host cell wall has a great influence on the viral entry mechanism and different cell types expose diverse external envelope structures. Three main entry strategies have been reported for viral entry in bacteria: genome release through an icosahedral vertex; dissociation of virion at the cell envelope; and virion penetration via membrane fusion (Poranen et al., 2002). Thus far insights into the mechanisms of entry by archaeal viruses have been based on coincidental observations. However, more recently a few detailed analyses have provided a better understanding of the molecular mechanisms at play in archaeal virus-host systems from geothermal environments.

\section{Interaction with Cellular Appendages}

Filamentous, flexible viruses of the Lipothrixviridae family have been classified into four different genera partly based on the virion core and terminal structures. Indeed, the exposed filaments can vary in number from one (AFV9, Acidianus filamentous virus 9) to six (SIFV, Sulfolobus islandicus filamentous virus) or even form complex structures like claws (AFV1) or brushes (AFV2; Arnold et al., 2000; Bettstetter et al., 2003; Haring et al., 2005b; Bize et al., 2008). The high diversity of terminal structures observed in this particular family strongly suggests their involvement in cellular adsorption processes. Indeed, AFV1 particles terminate with claws that mediate attachment to cellular pili (Bettstetter et al., 2003). In the case of AFV2, the "bottle brush," a complex collar termini with two sets of filaments, should be able to interact with the surface of host cells directly since its specific host doesn't show any extracellular appendages (Haring et al., 2005b). In addition, SIFV virions display mop-like structures found in open or closed conformations (Arnold et al., 2000). Hence, lipothrixviruses are decorated with diverse and unique terminal structures that play a major role in recognition and interaction with the host cell.

In a similar manner, the stiff, filamentous rudivirus SIRV2 (Sulfolobus islandicus rod-shaped virus 2) was also shown to bind host pili by the three terminal fibers of virions. SIRV2 is one of the more appealing models to study virus-host interactions in archaea (Prangishvili et al., 2013). Recently published analyses concluded that adsorption occurs within the first minute of infection, much more efficiently than in halophilic archaeal systems for which binding requires several hours (Kukkaro and Bamford, 2009). The particles of SIRV2 specifically attach to the tip of host pili-like structures leading to a strong and irreversible interaction between the viral and cellular determinants (Figure 1A). Subsequently, viruses are found on the side of the appendages indicating a progression toward the cell surface where DNA entry is concomitant with virion disassembly (Quemin et al., 2013; Figures 1C,D). Thus, the three fibers located at the virion termini represent the viral antireceptors involved in recognition of host cells and are responsible for the primary adsorption (Figure 1B). It is noteworthy that both ends of the virions have an equal binding capacity as previously noticed for the lipothrixvirus AFV1 (Bettstetter et al., 2003). The families Lipothrixviridae and Rudiviridae belong to the order Ligamenvirales and are known to attach to extracellular filaments (Prangishvili and Krupovic, 2012). Although AFV1 is capable of binding the side of host pili, a feature shared with bacterial leviviruses, cystoviruses and some tailed bacteriophages (Poranen et al., 2002), the interaction of SIRV2 with Sulfolobus filaments occurs initially via the tip. This resembles more closely the primary adsorption observed in the inoviruses (Rakonjac et al., 2011). All these data suggest that linear archaeal viruses employ a common strategy for the initiation of infection although 


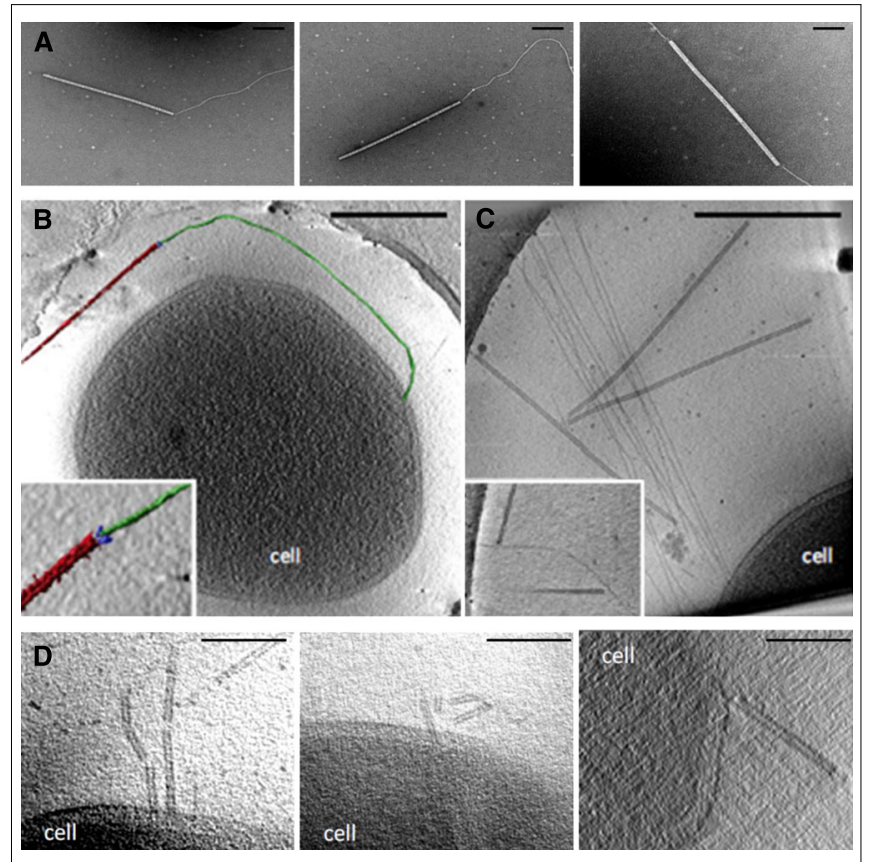

FIGURE 1 | Entry of SIRV2 in S. islandicus LAL14/1 cells.

(A) Transmission electron micrographs showing that SIRV2 virions interact with purified cellular filaments. Stained with $2 \%$ uranyl acetate for $2 \mathrm{~min}$. Scale bar, $200 \mathrm{~nm}$. Electron micrographs of SIRV2 interaction with S. islandicus LAL14/1 cells. Samples were collected 1 min post-infection and flash-frozen for electron cryotomography (cryo-ET). The virions interact both at the filament tips (B) and along the length of the filaments (C). The lower left panel (B) also shows a segmented tomographic volume of the SIRV2 virion (red) attached to the tip of an S. islandicus filament (green). The three terminal virion fibers that appear to mediate the interaction are shown in blue (the inset depicts a magnified view of the interaction between the virion fibers and the tip of the filament). The inset in the lower right panel (C) depicts two virions bound to the sides of a single filament. Scale bars, $500 \mathrm{~nm}$. (D) Tomographic slices through $S$. islandicus LAL14/1 cells at 1 min after infection with SIRV2 reveals partially disassembled SIRV2 virions at the cell surface. Adapted from (Quemin et al., 2013). Scale bar, $100 \mathrm{~nm}$.

the molecular mechanisms involved are most likely to be distinct.

\section{Interaction with Cell Surface}

As a general rule, viral entry implies direct or indirect binding to the cell surface depending on whether a primary adsorption step is required. In the case of SIRV2, analysis of virus-resistant strains provided interesting candidates for the receptors of SIRV2 virions at the cell surface. In fact, two operons were identified: sso23862387 and sso3139-3141 (Deng et al., 2014). The former encodes proteins homologous to components of type IV pili and the latter presumably a membrane-associated cell surface complex. In S. acidocaldarius, the assembly ATPase, AapE, and the central membrane protein, AapF, homologous to Sso2386 and Sso2387, respectively, are both essential for the assembly of the type IV adhesive pilus (Henche et al., 2012). The sso3139-3141 operon is thought to encode a membrane bound complex, which could function as a secondary receptor for SIRV2 (Deng et al., 2014).

While entry of rudiviruses, and filamentous archaeal viruses in general, relies on two coordinated adsorption steps, other systems interact spontaneously with the cell surface. As far back as 1984, SSV1 (Sulfolobus spindle-shaped virus 1) was reported to exist in different states: isolated particles, incorporated in typical rosette-like aggregates or even bound to cell-derived membrane (Martin et al., 1984). The best known member of the Fuselloviridae family displays a lemon-shaped morphotype with terminal fibers at one of the two pointed ends (Stedman et al., 2015). The set of short, thin filaments of the $\alpha$-fuselloviruses are involved in viral attachment and association with hostderived structures in general. However, the $\beta$-fuselloviruses, SSV6 and ASV1 (Acidianus spindle-shaped virus 1), exhibit more pleomorphic virions with three or four thick, slightly curved fibers (Krupovic et al., 2014). Although these appendages do not interact with each other as observed for SSV1, some genomic features strongly suggest that the fibers are composed of host-attachment proteins (Redder et al., 2009). Notably, one gene common to all family members (SSV1_C792) and two genes in $\beta$-fuselloviruses (SSV6_C213 and SSV6_B1232) encode for the protein responsible for terminal fibers. This protein shares a similar fold with the adsorption protein P2 of bacteriophage PRD1 (Grahn et al., 2002; Redder et al., 2009). In addition, the pointed end of the enveloped virus ABV (Acidianus bottle-shaped virus), from the Ampullaviridae family, is involved in attachment to membrane vesicles and formation of virion aggregates (Haring et al., 2005a). Therefore, even if data are still scarce, interaction with cellular membranes appears to be a common feature of hyperthermophilic archaeal viruses that contain a lipidic envelope. This particularly interesting feature merits further investigation.

\section{Release of Viral Genome}

Receptor recognition and binding typically induce a cascade of events that start with structural reorganization of the virions and lead to viral genome penetration through the cell envelope (Dimitrov, 2004). Non-enveloped viruses either inject the genome into the cell interior while leaving the empty capsid associated with the cell envelope or deliver the nucleic acids concomitantly with disassembly of the virion at the cell surface. Superficially, the entry of SIRV2 is similar to that of Ff inoviruses or flagellotrophic phages, which bind F-pili and flagella respectively (Guerrero-Ferreira et al., 2011; Rakonjac et al., 2011). First, the interaction with host pili-like structures has been shown and secondly, partially broken particles have been observed at the cellular membrane (Quemin et al., 2013; Figure 1). Notably, no archaeal retraction pili has been identified so far and flagella (called archaella in archaea) of Sulfolobus are considerably thicker than the filaments to which SIRV2 binds (Lassak et al., 2012). Additional experiments are needed in order to determine whether the mechanisms of SIRV2 translocation and genome delivery are related to those employed by $\mathrm{Ff}$ inoviruses and flagellotrophic bacteriophages, or are completely novel.

Lipid-containing viruses display unusual virion architecture and appear to make direct contact with the plasma membrane. It is reasonable to assume that enveloped viruses rely on a fundamentally different entry mechanism to that employed by non-enveloped filamentous viruses, such as rudiviruses. They might deliver their genetic material into the cell interior by fusion between the cytoplasmic membrane and the viral 
envelope in a similar fashion to the eukaryotic enveloped viruses (Vaney and Rey, 2011). ATV (Acidianus two-tailed virus) resembles fuselloviruses with virions extruded from host cells as lemon-shaped. However, ATV has been classified within the Bicaudaviridae partly due to its peculiar life cycle (Haring et al., 2005c). Surprisingly, at temperatures close to that of its natural habitat $\left(85^{\circ} \mathrm{C}\right)$, the released tail-less particles show the formation of two long tails protruding from the pointed ends. These extracellular developed tubes contain a thin filament inside and terminate in an anchor-like structure, not observed in the tail-less progeny. The two virion forms, tail-less and two-tailed, were reported to be infectious, thereby indicating that the termini are not involved in the initial stages of infection (Prangishvili et al., 2006b). However, genomic analysis as well as molecular studies highlighted some viral encoded proteins that could be important during infection. For example, the three largest open reading frames (ORFs) and one of the CPs have putative coiled-coil domains, which are usually associated with specific protein-protein interactions and protein complex formation. Moreover, two other proteins carry proline-rich regions (ORF567 and ORF1940) similar to the protein TPX and are abundant during infection by lipothrixvirus TTV1 (Thermoproteus tenax virus 1; Neumann and Zillig, 1990). Notably, in particular the motif TPTP has been implicated in host protein recognition for the African swine fever virus (Kay-Jackson, 2004). Finally, pull-down experiments provided evidence for a strong interaction between the ATV protein P529 and OppAss as well as cellular Sso1273, encoding a viral AAA ATPase. The cellular OppAss, an $\mathrm{N}$-linked glycoprotein, is most likely part of the binding components of the $\mathrm{ABC}$ transporter system. It is encoded within the same operon and could serve as a receptor. It has also been proposed that the AAA ATPase would trigger ATV host cell receptor recognition. This is based on the hypothetical requirement of its endonuclease activity for the cleavage of the circular viral DNA prior to entry in the cell (Erdmann et al., 2011).

The case of the bottle-shaped virus ABV is also particularly intriguing. The enveloped particles display an elaborate organization with a funnel-shaped body composed of the "stopper," the nucleoprotein core and the inner core. Presumably, the so-called "stopper" takes part in binding to the cellular receptor and is the only component to which the viral genome is directly attached. Therefore, it has been suggested that the "stopper" could play the role of an "injection needle" in a manner similar to that found in bacterial viruses. Actually, it is well known that head-tail bacteriophages belonging to the Caudovirales order use this transmembrane pathway for channeling and delivery of nucleic acids (Poranen et al., 2002). The inner core of ABV virions is the most labile part and could undergo structural changes that would facilitate the release of viral DNA (Haring et al., 2005a). Whether the energy accumulated in the structure after packaging of the supercoiled nucleoprotein is sufficient to transport the whole genetic material into the cytoplasm is unclear. However, relaxation of the nucleoprotein filament, wound up as an inverse cone, concomitantly with its funneling into the cell could be an efficient way of utilizing the energy stored during packaging for DNA injection as previously observed in bacteria (Poranen et al., 2002).
How archaeal viruses interact with the cell surface and deliver the viral genome into the host cytoplasm is still puzzling. Some systems, rudiviruses and lipothrixviruses, show similarities to their bacterial counterparts while others, fuselloviruses, bicaudavirus and ampullavirus, could be related to eukaryotic viruses. Identification of the pathways utilized by both filamentous and unique lipid-containing viruses represents a great challenge and one of the main issues that should be tackled in the near future. It is noteworthy that the S-layer is generally composed of heavily glycosylated proteins and many archaeal viruses exhibit glycosylated capsid proteins. The fact that several glycosyltransferases are encoded in viral genomes (Krupovic et al., 2012) is particularly intriguing. Indeed, protein glycosylation is an important process, which could be involved in virion stability and/or interaction with the host cell (Markine-Goriaynoff et al., 2004; Meyer and Albers, 2013).

\section{Strategies for Viral Escape from the Host Cell}

The last and essential step of the viral infection cycle is escape of viral particles from the host cell. So far, the egress mechanism has been analyzed for only a small subset of archaeal viruses (Torsvik and Dundas, 1974; Bize et al., 2009; Brumfield et al., 2009; Snyder et al., 2013a). Some viruses are completely lytic, while others are apparently stably produced without causing evident cell lysis (Bettstetter et al., 2003). In addition, there are temperate archaeal viruses with a lysogenic life cycle for which induction of virion production in some cases leads to cell disruption (Janekovic et al., 1983; Schleper et al., 1992; Prangishvili et al., 2006b).

The release mechanisms utilized by archaeal viruses can be divided in two categories: those for which the cell membrane is disrupted and those where the membrane integrity remains intact. The strategy for egress is linked with the assembly mechanism of new virions. Some archaeal viruses are known to mature inside the cell cytoplasm and provoke lysis, such as STIV1 (Sulfolobus turreted icosahedral virus) and SIRV2 (Bize et al., 2009; Brumfield et al., 2009; Fu et al., 2010). However, most non-lytic viruses undergo final maturation concomitantly with passage through the cell membrane (Roine and Bamford, 2012) or even in the extracellular environment, as observed for ATV (Haring et al., 2005c).

\section{Cell Membrane Disruption \\ Lysis by Complete Membrane Disruption}

Disruption of cell membranes can be caused by lytic or temperate viruses. In case of temperate viruses the cell lysis occurs typically after induction of virus replication and virion formation. Virion production of lysogenic viruses can be induced by various stimuli such as; UV radiation, addition of mitomycin C, starvation or shift from aerobic to anaerobic growth (Janekovic et al., 1983; Schleper et al., 1992; Prangishvili et al., 2006b; Mochizuki et al., 2011).

The first archaeal viruses were isolated from hypersaline environments long before archaea were recognized as a separate domain of life (Torsvik and Dundas, 1974; Wais et al., 1975). These viruses infect halophiles, which belong to the phylum 
Euryarchaeota. The viral particles exhibit a head-and-tail morphology classical for bacterial viruses. Infection with these viruses resulted in complete lysis of the cells, suggested by a decrease in culture turbidity. Later on, more euryarchaeal viruses were isolated from hypersaline or anaerobic environments, and several of these viruses displayed non-head-tail morphologies such as icosahedral or spindle shapes. Again, in some cases, optical density diminishes with time after viral infection, indicating that a part of these viruses initiate cell lysis (Bath and Dyall-Smith, 1998; Porter et al., 2005; Jaakkola et al., 2012). However, several euryarchaeal viruses apparently do not cause cell lysis.

Amongst hyperthermophilic crenarchaeal viruses there has only been a single report of a decrease in the turbidity of infected cultures (Prangishvili et al., 2006a). In this case, induction of virion production of the lysogenic viruses TTV1-3 led to cell lysis, which was measured by decreasing turbidity (Janekovic et al., 1983). Lysis induced by archaeal viruses can either be coupled with virion production (Jaakkola et al., 2012), or take place after the largest virion burst, therefore raising the possibility of an additional release mechanism in such systems (Bath and DyallSmith, 1998; Porter et al., 2005, 2013). Although measurement of optical density is a classical method for the characterization of viral cycles and decrease in turbidity has been observed for several archaeal viruses, no molecular mechanism to achieve complete membrane disruption in archaea has been proposed as yet.

Bacterial virus-host systems are widely studied and as a result the mechanism of lysis used by bacterial viruses is better understood. Bacterial viruses typically induce cell lysis by degradation of the cell wall, which is achieved by muralytic endolysins (Young, 2013). In addition, most bacterial viruses encode small proteins named holins (Bernhardt et al., 2001a,b; Catalao et al., 2013). Holins usually accumulate harmlessly in the bacterial cell membrane until a critical concentration is reached and nucleation occurs. Nucleation results in formation of two dimensional aggregates, "holin rafts," that rapidly expand and create pores in lipid layers through which the endolysins can reach the cell wall (Young, 2013). In gram negative bacteria the presence of an outer membrane requires additional virus-encoded proteins, spanins, which are suggested to induce fusion of the inner and outer membrane (Berry et al., 2012). After an initial degradation of the peptidoglycan cell wall, the cells burst due to osmotic pressure, explaining total loss of turbidity observed for infected bacterial cultures (Berry et al., 2012). Accurate timing of lysis is essential for successful virus reproduction and is achieved by regulation of holin expression (Young, 2013). Since archaea lack a peptidoglycan cell wall, endolysinholin egress systems are not effective in archaea. Only a few archaeal species contain a peptidoglycan-like cell wall consisting of pseudomurein polymers (Albers and Meyer, 2011). The oligosaccharide backbone and amino acid interbridges of murein and pseudomurein are different, rendering bacterial endolysins ineffective to pseudomurein (Visweswaran et al., 2011). However, pseudomurein degrading enzymes are encoded by a few archaeal viruses infecting methanogens; the integrated provirus $\psi \mathrm{M} 100$ from Methanothermobacter wolfeii and the virus $\psi \mathrm{M} 1$ infecting M. marburgensis (Luo et al., 2001). How these intracellularly produced viral endolysis traverse the archaeal cell membrane in order to degrade the pseudomurein cell wall is not clear, since the mandatory pore forming holins have not been identified in the genomes of these viruses. The possible presence of archaeal holins could be currently overlooked, as genes encoding holins share generally very little sequence similarity, making it difficult to predict their presence in genomes (Saier and Reddy, 2015).

The large majority of archaea lack a pseudomurein cell wall. Therefore instead of a endolysin-holin system, a fundamentally different lysis mechanism would be required for release of virions from these cell wall lacking archaea. One hypothesis is that archaeal viruses employ holins to disrupt the cell membrane, possibly combined with proteolytic enzymes in order to degrade the S-layer. To date there are about a dozen holin homologs identified in archaeal genomes based on sequence similarity (Reddy and Saier, 2013), but none of the predicted proteins have been tested in vivo. Moreover, not a single holin-encoding gene has been identified in the genomes of currently isolated archaeal viruses (Reddy and Saier, 2013; Saier and Reddy, 2015). In addition, specific enzymes capable of S-layer degradation are currently unknown and S-layer proteins and sugars display a large diversity in different species (Albers and Meyer, 2011). Thus in contrast to bacterial endolysins that degrade peptidoglycan cell walls of virtually all bacteria, specific tailor made proteases would be required to degrade archaeal S-layers of different species.

\section{Lysis by Formation of Defined Apertures}

The egress mechanism of only two archaeal viruses (STIV1 and SIRV2) has been studied in high molecular detail. Both employ a release mechanism that relies on the formation of pyramidal shaped egress structures, which are unique to archaeal systems (Bize et al., 2009; Brumfield et al., 2009; Quax et al., 2011; Snyder et al., 2011). At first glance, both viruses were regarded as nonlytic viruses, since a decrease in cell culture turbidity was never observed (Prangishvili et al., 1999; Rice et al., 2004). However, the use of several electron microscopy techniques clearly showed that the two viruses induced cell lysis (Bize et al., 2009; Brumfield et al., 2009). Their particular lysis mechanism yields empty cell ghosts explaining the maintenance of culture turbidity.

Infection by SIRV2 and STIV1 leads to formation of several pyramidal shaped structures on the cell membrane of $S$. islandicus and S. solfataricus respectively (Bize et al., 2009; Brumfield et al., 2009; Prangishvili and Quax, 2011; Figure 2A). These virus-associated pyramids (VAPs) exhibit sevenfold rotational symmetry and protrude trough the S-layer (Quax et al., 2011; Snyder et al., 2011; Figures 2B-D). At the end of the infection cycle, the seven facets of the VAPs open outward, generating large apertures through which assembled virions exit from the cell (Fu et al., 2010; Quax et al., 2011; Daum et al., 2014; Figure 2B). The baseless VAP consist of multiple copies of a $10 \mathrm{kDa}$ viral encoded protein, PVAP (STIV1_C92/SIRV2_P98) (Quax et al., 2010; Snyder et al., 2013a). This protein contains a transmembrane domain, but lacks a signal sequence and seems to be inserting in membranes based on hydrophobicity of its transmembrane domain (Quax et al., 2010; Daum et al., 2014). PVAP has the remarkable property to form pyramidal structures in virtually all biological membranes, as was demonstrated by heterologos 

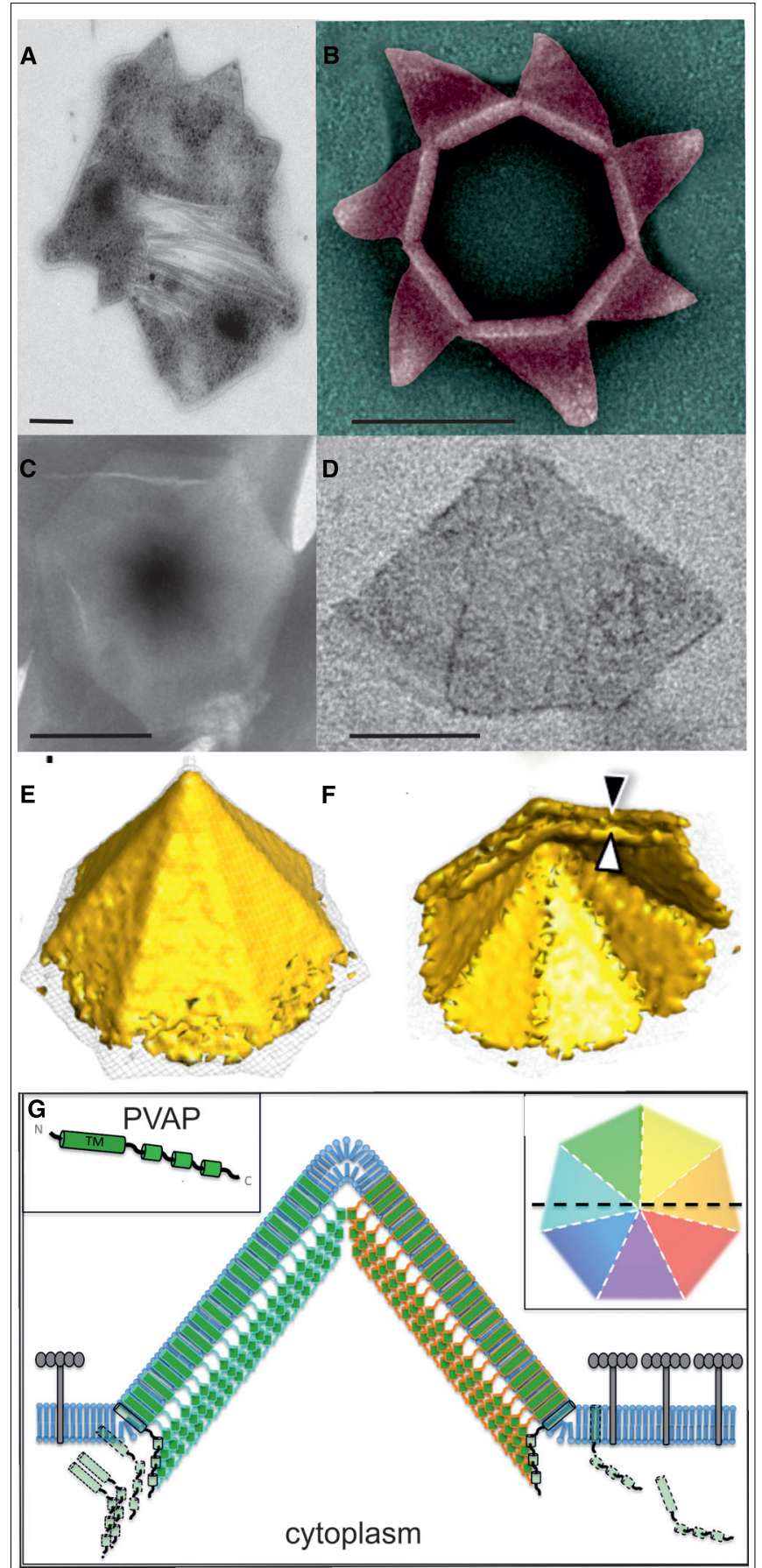

FIGURE 2 | Remarkable archaeal virion egress structure. (A) Scanning electron micrograph of an SIRV2 infected S. islandicus cell displaying several VAPs. (B) Transmission electron micrographs of isolated VAPs in closed and (C) open conformation. (D) Solid representation of VAP obtained by subtomogram averaging displaying the (E) outside and (F) interior. (G) Model of VAP formation. Adapted from (Bize et al., 2009; Quax et al., 2011; Daum et al., 2014). Scale bar, $100 \mathrm{~nm}$.

expression of PVAP in archaea, bacteria and eukaryotes (Quax et al., 2011; Snyder et al., 2013a; Daum et al., 2014).

Nucleation of the PVAP-induced structure starts on the cell membrane, most likely with the formation of a heptamer of
PVAP subunits (Daum et al., 2014). The structures develop by the outward expansion of their seven triangular facets. They reach sizes of up to $200 \mathrm{~nm}$ in diameter, both in natural and heterologous systems (Quax et al., 2011; Daum et al., 2014). In contrast to bacterial holin rafts, the formation of VAPs is not a sudden process depending on a critical protein concentration. PVAP transcripts steadily increase throughout the infection cycle and PVAP integrates in the membrane until late stages of infection (Quax et al., 2010, 2013; Maaty et al., 2012). Although VAPs are slowly formed, their actual opening is quite rapid (Bize et al., 2009; Brumfield et al., 2009; Snyder et al., 2011; Daum et al., 2014). The nature of the signal triggering this opening has not been identified yet. VAPs, formed after heterologous PVAP expression, in bacteria and eukaryotes were never observed in open conformation, suggesting that an archaeal specific factor is required (Daum et al., 2014). It has been proposed that the archaeal ESCRT (Endosomal Sorting Complex Required for Transport) machinery could be involved in the STIV1 VAP-based exit (Snyder et al., 2013b). Considering that genes encoding ESCRT machinery are specifically down regulated during SIRV2 infection (Quax et al., 2013), and that STIV1 contains in contrast to SIRV2 an inner lipid layer (Veesler et al., 2013), STIV1 requirement of the ESCRT system might be independent from VAP-induced lysis.

The ultrastructure of VAPs of SIRV2 was studied by whole cell cryo-tomography and subtomogram averaging. This revealed the presence of two layers, of which the outer one is continuous with the cell membrane and presumably formed by the N-terminal transmembrane domain (Daum et al., 2014; Figures 2E,F). The inner layer represents a protein sheet formed by tight protein-protein interactions of the C-terminal domain of the protein (Daum et al., 2014). The strong interactions between PVAP monomers are suggested to exclude most lipids and membrane proteins from the VAP assembly site, in a similar fashion as holin raft formation (White et al., 2011; Figure 2G). $\mathrm{S}$-layer proteins are anchored in the membrane, and consequently will be excluded from the VAP assembly site, providing a strategy for VAP protrusion through the S-layer.

The described VAP-based egress mechanism is archaeal specific. Homologues of PVAP are only found amongst some archaeal viruses (Quax et al., 2010). However, the majority of archaeal viruses lack PVAP, suggesting that they rely on a different and as yet unknown mechanism for egress.

\section{Viral Extrusion without Membrane Disruption}

While the first isolated archaeal viruses were lytic, subsequent characterization of more viruses revealed that the large majority do not cause lysis of the host cell. To date, lytic viruses make up half of the viruses infecting euryarchaeota, and only three in crenarchaea (Torsvik and Dundas, 1974; Wais et al., 1975; Janekovic et al., 1983; Bize et al., 2009; Brumfield et al., 2009; Pina et al., 2011). In addition, some studies indicate that free virions can be observed before disruption of archaeal cells, suggesting that another egress mechanism exists, which preserves cell membrane integrity. It might be possible that some lytic archaeal viruses have been currently overlooked due to special characteristics of their lysis mechanism, as was the case for STIV1 and SIRV2 (Prangishvili et al., 1999; Rice et al., 2004). Nevertheless, the low 
number of lytic archaeal viruses contrasts with the situation in bacteria, for which lytic viruses are very common. The majority of archaeal viruses are thought to be continuously produced without integrating into the host genome or killing their hosts (Pina et al., 2014). This equilibrium between viruses and cells is referred to as a "stable carrier state" (Bettstetter et al., 2003; Prangishvili and Garrett, 2005; Prangishvili et al., 2006a). The nature of this stable carrier state and the mechanisms by which virions are extruded from archaea without causing cell lysis, remain poorly understood.

In contrast to the situation in archaea, the majority of bacterial viruses are lytic. Almost all bacterial viruses exit via the holin based mechanism described above. However, an exception to the rule are the bacterial filamentous viruses belonging to the Inoviridae that egress without causing cell lysis (Rakonjac et al., 2011). The majority of the inoviruses infect gram negative bacteria. Assembly of inoviruses is finalized during particle extrusion. The interaction between the packaging signal of the viral genome and the cellular membrane initiates the exit step (Russel and Model, 1989). Virally encoded proteins are thought to form pores in the inner membrane through which the DNA is extruded. Multiple copies of the major CP accumulate in the inner membrane and associate with the ssDNA viral genome while it is passing through the virus-induced pores (Rakonjac et al., 1999). A barrel-like structure in the outer membrane permits the release of progeny and is composed of multiple copies of a virusencoded protein with homology to proteins of type II secretion systems and type IV pili (Marciano et al., 2001). Alternatively, other inoviruses use the host secretion machinery to traverse the outer membrane (Davis et al., 2000; Bille et al., 2005). Even though replication of the viral genome and constituents might burden the cell, the infection of inoviruses does not lead to cell death and is a continuous process. There are several archaeal filamentous viruses known. However, filamentous archaeal viruses are not related to the bacterial inoviruses, nor encode homologs of the secretion-like proteins involved in egress of inoviruses (Janekovic et al., 1983; Bize et al., 2009; Quax et al., 2010; Pina et al., 2014). Therefore the filamentous archaeal viruses must rely on an alternative mechanism for viral extrusion from the cell.

Interestingly, lipid-containing archaeal viruses are quite common (Roine and Bamford, 2012). There are some archaeal icosahedral viruses that possess an inner membrane, such as STIV and SH1 (Bamford et al., 2005; Khayat et al., 2005; Porter et al., 2005). In addition, the filamentous lipothrixviruses (Janekovic et al., 1983; Arnold et al., 2000; Bettstetter et al., 2003), the spherical virus PSV (Pyrobaculum spherical virus; Haring et al., 2004) and the pleiomorphic euryarchaeal viruses (Pietila et al., 2009, 2013) all contain an external lipid envelope. The lipids are typically derived from the host cell. Several eukaryotic viruses contain a membrane that is usually obtained during "budding," a process by which particles egress without disturbing

\section{References}

Albers, S. V., and Meyer, B. H. (2011). The archaeal cell envelope. Nat. Rev. Microbiol. 9, 414-426. doi: 10.1038/nrmicro2576 the membrane integrity. Eukaryotic enveloped viruses either encode their own scission proteins, or hijack vesicle formation machinery of their host (Rossman and Lamb, 2013). Archaea are also reported to produce vesicles (Soler et al., 2008; Ellen et al., 2011), and the machinery responsible for vesicle production might be utilized by lipid envelope containing viruses in archaea as well. In particular, the pleiomorphic viruses infecting euryarchaea are likely to be released through budding as their envelope has the same lipid composition as the host they infect (Pietila et al., 2009; Roine et al., 2010).

The most common scission machinery employed by eukaryotic viruses is the ESCRT system (Votteler and Sundquist, 2013). In eukaryotes these proteins are responsible for endosomal sorting in the multi vesicular body. Well-characterized viruses such as Ebola and human immunodeficiency virus (HIV) use the ESCRT proteins during egress (Harty et al., 2000; Weissenhorn et al., 2013). Interestingly, proteins homologous to ESCRT components have been identified in several archaea, where they are involved in cell division (Lindas et al., 2008; Samson et al., 2008; Makarova et al., 2010; Pelve et al., 2011). These proteins represent potential players in budding-like extrusion processes in archaea. The mechanism underlying the release of temperate archaeal viruses remains largely unexplored and represents an appealing area of research that should shed light on original and unconventional strategies.

\section{Concluding Remarks}

The last few years have shown a steady increase in an understanding of archaeal virus-host interactions, therefore revealing the first insights into viral interactions with the archaeal membrane. Viruses have developed various strategies to cross the membrane. These strategies are adapted to the nature of the cell envelope of their host. Some archaeal viruses employ fascinating novel mechanisms, while others appear to rely on processes that at first sight are analogous to their bacterial counterparts. Additional research will help to determine to which extent bacterial, eukaryotic and archaeal virospheres are evolutionary related. The uniqueness of the archaeal cell surface, and the diversity of the currently described archaeal entry and egress mechanisms, argue in favor of future discovery of more innovative and surprising molecular mechanisms.

\section{Acknowledgments}

We thank Dr. D. Prangishvili and Dr. S. Gill for critical reading of the manuscript, useful suggestions and comments. This work was supported by l'Agence Nationale de la Recherche, project "EXAVIR," by a FWO Pegasus Marie Curie fellowship to TQ. and by a grant from the French government and the Université Pierre et Marie Curie, Paris VI to EQ.

Arnold, H. P., Zillig, W., Ziese, U., Holz, I., Crosby, M., Utterback, T., et al. (2000). A novel lipothrixvirus, SIFV, of the extremely thermophilic crenarchaeon Sulfolobus. Virology 267, 252-266. doi: 10.1006/viro.1999. 0105 
Bamford, D. H., Ravantti, J. J., Ronnholm, G., Laurinavicius, S., Kukkaro, P., Dyall-Smith, M., et al. (2005). Constituents of SH1, a novel lipid-containing virus infecting the halophilic euryarchaeon Haloarcula hispanica. J. Virol. 79, 9097-9107. doi: 10.1128/jvi.79.14.9097-9107.2005

Bath, C., and Dyall-Smith, M. L. (1998). His1, an archaeal virus of the Fuselloviridae family that infects Haloarcula hispanica. J. Virol. 72, 9392-9395.

Bergh, O., Borsheim, K. Y., Bratbak, G., and Heldal, M. (1989). High abundance of viruses found in aquatic environments. Nature 340, 467-468. doi: $10.1038 / 340467 \mathrm{a} 0$

Bernhardt, T. G., Struck, D. K., and Young, R. (2001a). The lysis protein E of phi $\mathrm{X} 174$ is a specific inhibitor of the MraY-catalyzed step in peptidoglycan synthesis. J. Biol. Chem. 276, 6093-6097. doi: 10.1074/jbc.M00763 8200

Bernhardt, T. G., Wang, I. N., Struck, D. K., and Young, R. (2001b). A protein antibiotic in the phage Qbeta virion: diversity in lysis targets. Science 292, 2326-2329. doi: 10.1126/science.1058289

Berry, J., Rajaure, M., Pang, T., and Young, R. (2012). The spanin complex is essential for lambda lysis. J. Bacteriol. 194, 5667-5674. doi: 10.1128/JB.01245-12

Bettstetter, M., Peng, X., Garrett, R. A., and Prangishvili, D. (2003). AFV1, a novel virus infecting hyperthermophilic archaea of the genus Acidianus. Virology 315, 68-79. doi: 10.1016/S0042-6822(03)00481-1

Bille, E., Zahar, J. R., Perrin, A., Morelle, S., Kriz, P., Jolley, K. A., et al. (2005). A chromosomally integrated bacteriophage in invasive meningococci. J. Exp. Med. 201, 1905-1913. doi: 10.1084/jem.20050112

Bize, A., Karlsson, E. A., Ekefjard, K., Quax, T. E., Pina, M., Prevost, M. C., et al. (2009). A unique virus release mechanism in the Archaea. Proc. Natl. Acad. Sci. U.S.A. 106, 11306-11311. doi: 10.1073/pnas.0901238106

Bize, A., Peng, X., Prokofeva, M., Maclellan, K., Lucas, S., Forterre, P., et al. (2008). Viruses in acidic geothermal environments of the Kamchatka Peninsula. Res. Microbiol. 159, 358-366. doi: 10.1016/j.resmic.2008.04.009

Borsheim, K. Y., Bratbak, G., and Heldal, M. (1990). Enumeration and biomass estimation of planktonic bacteria and viruses by transmission electron microscopy. Appl. Environ. Microbiol. 56, 352-356.

Brumfield, S. K., Ortmann, A. C., Ruigrok, V., Suci, P., Douglas, T., and Young, M. J. (2009). Particle assembly and ultrastructural features associated with replication of the lytic archaeal virus Sulfolobus turreted icosahedral virus. J. Virol. 83, 5964-5970. doi: 10.1128/jvi.02668-08

Catalao, M. J., Gil, F., Moniz-Pereira, J., Sao-Jose, C., and Pimentel, M. (2013). Diversity in bacterial lysis systems: bacteriophages show the way. FEMS Microbiol. Rev. 37, 554-571. doi: 10.1111/1574-6976.12006

Ceballos, R. M., Marceau, C. D., Marceau, J. O., Morris, S., Clore, A. J., and Stedman, K. M. (2012). Differential virus host-ranges of the Fuselloviridae of hyperthermophilic Archaea: implications for evolution in extreme environments. Front. Microbiol. 3:295. doi: 10.3389/fmicb.2012.00295

Daum, B., Quax, T. E., Sachse, M., Mills, D. J., Reimann, J., Yildiz, O., et al. (2014). Self-assembly of the general membrane-remodeling protein PVAP into sevenfold virus-associated pyramids. Proc. Natl. Acad. Sci. U.S.A. 111, 3829-3834. doi: 10.1073/pnas.1319245111

Davis, B. M., Lawson, E. H., Sandkvist, M., Ali, A., Sozhamannan, S., and Waldor, M. K. (2000). Convergence of the secretory pathways for cholera toxin and the filamentous phage, CTXphi. Science 288, 333-335. doi: 10.1126/science.288.5464.333

Deng, L., He, F., Bhoobalan-Chitty, Y., Martinez-Alvarez, L., Guo, Y., and Peng, X. (2014). Unveiling cell surface and type IV secretion proteins responsible for archaeal Rudivirus entry. J. Virol. 88, 10264-10268. doi: 10.1128/jvi.01495-14

Dimitrov, D. S. (2004). Virus entry: molecular mechanisms and biomedical applications. Nat. Rev. Microbiol. 2, 109-122. doi: 10.1038/nrmicro817

Ellen, A. F., Rohulya, O. V., Fusetti, F., Wagner, M., Albers, S. V., and Driessen, A. J. (2011). The sulfolobicin genes of Sulfolobus acidocaldarius encode novel antimicrobial proteins. J. Bacteriol. 193, 4380-4387. doi: 10.1128/jb. 05028-11

Erdmann, S., Scheele, U., and Garrett, R. A. (2011). AAA ATPase p529 of Acidianus two-tailed virus ATV and host receptor recognition. Virology 421, 61-66. doi: 10.1016/j.virol.2011.08.029

Fagan, R. P., and Fairweather, N. F. (2014). Biogenesis and functions of bacterial S-layers. Nat. Rev. Microbiol. 12, 211-222. doi: 10.1038/nrmicro3213

Fu, C. Y., Wang, K., Gan, L., Lanman, J., Khayat, R., Young, M. J., et al. (2010). In vivo assembly of an archaeal virus studied with whole-cell electron cryotomography. Structure 18, 1579-1586. doi: 10.1016/j.str.2010.10.005
Grahn, A. M., Daugelavicius, R., and Bamford, D. H. (2002). The small viral membrane associated protein $\mathrm{P} 32$ is involved in bacteriophage PRD1 DNA entry. J. Virol. 76, 4866-4872. doi: 10.1128/jvi.76.10.4866-4872. 2002

Guerrero-Ferreira, R. C., Viollier, P. H., Ely, B., Poindexter, J. S., Georgieva, M., Jensen, G. J., et al. (2011). Alternative mechanism for bacteriophage adsorption to the motile bacterium Caulobacter crescentus. Proc. Natl. Acad. Sci. U.S.A. 108, 9963-9968. doi: 10.1073/pnas.1012388108

Haring, M., Peng, X., Brugger, K., Rachel, R., Stetter, K. O., Garrett, R. A., et al. (2004). Morphology and genome organization of the virus PSV of the hyperthermophilic archaeal genera Pyrobaculum and Thermoproteus: a novel virus family, the Globuloviridae. Virology 323, 233-242. doi: 10.1016/j.virol.2004.03.002

Haring, M., Rachel, R., Peng, X., Garrett, R. A., and Prangishvili, D. (2005a). Viral diversity in hot springs of Pozzuoli, Italy, and characterization of a unique archaeal virus, Acidianus bottle-shaped virus, from a new family, the Ampullaviridae. J. Virol. 79, 9904-9911. doi: 10.1128/JVI.79.15.9904-9911. 2005

Haring, M., Vestergaard, G., Brugger, K., Rachel, R., Garrett, R. A., and Prangishvili, D. (2005b). Structure and genome organization of AFV2, a novel archaeal lipothrixvirus with unusual terminal and core structures. J. Bacteriol. 187, 3855-3858. doi: 10.1128/JB.187.11.3855-3858.2005

Haring, M., Vestergaard, G., Rachel, R., Chen, L., Garrett, R. A., and Prangishvili, D. (2005c). Virology: independent virus development outside a host. Nature 436, 1101-1102. doi: 10.1038/4361101a

Harty, R. N., Brown, M. E., Wang, G., Huibregtse, J., and Hayes, F. P. (2000). A PPxY motif within the VP40 protein of Ebola virus interacts physically and functionally with a ubiquitin ligase: implications for filovirus budding. Proc. Natl. Acad. Sci. U.S.A. 97, 13871-13876. doi: 10.1073/pnas.250277297

Henche, A. L., Ghosh, A., Yu, X., Jeske, T., Egelman, E., and Albers, S. V. (2012). Structure and function of the adhesive type IV pilus of Sulfolobus acidocaldarius. Environ. Microbiol. 14, 3188-3202. doi: 10.1111/j.1462-2920.2012.02898.x

Jaakkola, S. T., Penttinen, R. K., Vilen, S. T., Jalasvuori, M., Ronnholm, G., Bamford, J. K., et al. (2012). Closely related archaeal Haloarcula hispanica icosahedral viruses HHIV-2 and SH1 have nonhomologous genes encoding host recognition functions. J. Virol. 86, 4734-4742. doi: 10.1128/jvi.06666-11

Janekovic, D., Wunderl, S., Holz, I., Zillig, W., Gierl, A., and Neumann, H. (1983). TTV1, TTV2 and TTV3, a family of viruses of the extremely thermophilic, anaerobic, sulfur reducing archaebacterium Thermoproteus tenax. Mol. Gen. Genet. 192, 39-45. doi: 10.1007/BF00327644

Kay-Jackson, P. C. (2004). The CD2v protein of African swine fever virus interacts with the actin-binding adaptor protein SH3P7. J. Gen. Virol. 85, 119-130. doi: 10.1099/vir.0.19435-0

Khayat, R., Tang, L., Larson, E. T., Lawrence, C. M., Young, M., and Johnson, J. E. (2005). Structure of an archaeal virus capsid protein reveals a common ancestry to eukaryotic and bacterial viruses. Proc. Natl. Acad. Sci. U.S.A. 102, 18944-18949. doi: 10.1073/pnas.0506383102

Krupovic, M., Quemin, E. R., Bamford, D. H., Forterre, P., and Prangishvili, D. (2014). Unification of the globally distributed spindle-shaped viruses of the Archaea. J. Virol. 88, 2354-2358. doi: 10.1128/JVI.02941-13

Krupovic, M., White, M. F., Forterre, P., and Prangishvili, D. (2012). Postcards from the edge: structural genomics of archaeal viruses. Adv. Virus Res. 82, 33-62. doi: 10.1016/B978-0-12-394621-8.00012-1

Kukkaro, P., and Bamford, D. H. (2009). Virus-host interactions in environments with a wide range of ionic strengths. Environ. Microbiol. Rep. 1, 71-77. doi: 10.1111/j.1758-2229.2008.00007.x

Lassak, K., Ghosh, A., and Albers, S. V. (2012). Diversity, assembly and regulation of archaeal type IV pili-like and non-type-IV pili-like surface structures. Res. Microbiol. 163, 630-644. doi: 10.1016/j.resmic.2012.10.024

Lindas, A. C., Karlsson, E. A., Lindgren, M. T., Ettema, T. J., and Bernander, R. (2008). A unique cell division machinery in the Archaea. Proc. Natl. Acad. Sci. U.S.A. 105, 18942-18946. doi: 10.1073/pnas.0809467105

Luo, Y., Pfister, P., Leisinger, T., and Wasserfallen, A. (2001). The genome of archaeal prophage PsiM100 encodes the lytic enzyme responsible for autolysis of Methanothermobacter wolfeii. J. Bacteriol. 183, 5788-5792. doi: 10.1128/jb.183.19.5788-5792.2001

Maaty, W. S., Steffens, J. D., Heinemann, J., Ortmann, A. C., Reeves, B. D., Biswas, S. K., et al. (2012). Global analysis of viral infection in an archaeal model system. Front. Microbiol. 3:411. doi: 10.3389/fmicb.2012.00411 
Makarova, K. S., Yutin, N., Bell, S. D., and Koonin, E. V. (2010). Evolution of diverse cell division and vesicle formation systems in Archaea. Nat. Rev. Microbiol. 8, 731-741. doi: 10.1038/nrmicro2406

Markine-Goriaynoff, N., Gillet, L., Van Etten, J. L., Korres, H., Verma, N., and Vanderplasschen, A. (2004). Glycosyltransferases encoded by viruses. J. Gen. Virol. 85, 2741-2754.

Marciano, D. K., Russel, M., and Simon, S. M. (2001). Assembling filamentous phage occlude pIV channels. Proc. Natl. Acad. Sci. U.S.A. 98, 9359-9364. doi: 10.1073/pnas.161170398

Martin, A., Yeats, S., Janekovic, D., Reiter, W.-D., Aicher, W., and Zillig W. (1984). SAV 1, a temperate u.v.-inducible DNA virus-like particle from the archaeabacterium Sulfolobus acidocaldarius isolate B12. EMBO J. 3, 21652168.

Meyer, B. H., and Albers, S. V. (2013). Hot and sweet: protein glycosylation in Crenarchaeota. Biochem. Soc. Trans. 41, 384-3892. doi: 10.1042/BST20120296

Mochizuki, T., Sako, Y., and Prangishvili, D. (2011). Provirus induction in hyperthermophilic archaea: characterization of Aeropyrum pernix spindleshaped virus 1 and Aeropyrum pernix ovoid virus 1. J. Bacteriol. 193, 5412-5419. doi: $10.1128 / \mathrm{jb} .05101-11$

Molineux, I. J., and Panja, D. (2013). Popping the cork: mechanisms of phage genome ejection. Nat. Rev. Microbiol. 11, 194-204. doi: 10.1038/nrmicro2988

Neumann, H., and Zillig, W. (1990). The TTV1-encoded viral protein TPX: primary structure of the gene and the protein. Nucleic Acids Res. 18, 195. doi: 10.1093/nar/18.1.195

Pelve, E. A., Lindas, A. C., Martens-Habbena, W., De La Torre, J. R., Stahl, D. A., and Bernander, R. (2011). Cdv-based cell division and cell cycle organization in the thaumarchaeon Nitrosopumilus maritimus. Mol. Microbiol. 82, 555-566. doi: 10.1111/j.1365-2958.2011.07834.x

Pietila, M. K., Laurinmaki, P., Russell, D. A., Ko, C. C., Jacobs-Sera, D., Butcher, S. J., et al. (2013). Insights into head-tailed viruses infecting extremely halophilic archaea. J. Virol. 87, 3248-3260. doi: 10.1128/jvi.03397-12

Pietila, M. K., Roine, E., Paulin, L., Kalkkinen, N., and Bamford, D. H. (2009). An ssDNA virus infecting archaea: a new lineage of viruses with a membrane envelope. Mol. Microbiol. 72, 307-319. doi: 10.1111/j.1365-2958.2009.06642.x

Pina, M., Basta, T., Quax, T. E., Joubert, A., Baconnais, S., Cortez, D., et al. (2014). Unique genome replication mechanism of the archaeal virus AFV1. Mol. Microbiol. 92, 1313-1325. doi: 10.1111/mmi.12630

Pina, M., Bize, A., Forterre, P., and Prangishvili, D. (2011). The archeoviruses. FEMS Microbiol. Rev. 35, 1035-1054. doi: 10.1111/j.1574-6976.2011.00280.x

Pohlschroder, M., Ghosh, A., Tripepi, M., and Albers, S. V. (2011). Archaeal type IV pilus-like structures-evolutionarily conserved prokaryotic surface organelles. Curr. Opin. Microbiol. 14, 357-363. doi: 10.1016/j.mib.2011.03.002

Poranen, M. M., Daugelavicius, R., and Bamford, D. H. (2002). Common principles in viral entry. Аnпu. Rev. Microbiol. 56, 521-538. doi: 10.1146/annurev.micro.56.012302.160643

Porter, K., Kukkaro, P., Bamford, J. K., Bath, C., Kivela, H. M., Dyall-Smith, M. L., et al. (2005). SH1: a novel, spherical halovirus isolated from an Australian hypersaline lake. Virology 335, 22-33. doi: 10.1016/j.virol.2005.01.043

Porter, K., Tang, S. L., Chen, C. P., Chiang, P. W., Hong, M. J., and Dyall-Smith, M. (2013). PH1: an archaeovirus of Haloarcula hispanica related to SH1 and HHIV-2. Archaea 2013, 456318. doi: 10.1155/2013/456318

Prangishvili, D. (2013). The wonderful world of archaeal viruses. Annu. Rev. Microbiol. 67, 565-585. doi: 10.1146/annurev-micro-092412-155633

Prangishvili, D., Arnold, H. P., Gotz, D., Ziese, U., Holz, I., Kristjansson, J. K., et al. (1999). A novel virus family, the Rudiviridae: structure, virus-host interactions and genome variability of the Sulfolobus viruses SIRV1 and SIRV2. Genetics 152, 1387-1396.

Prangishvili, D., Forterre, P., and Garrett, R. A. (2006a). Viruses of the Archaea: a unifying view. Nat. Rev. Microbiol. 4, 837-848. doi: 10.1038/nrmicro1527

Prangishvili, D., Vestergaard, G., Haring, M., Aramayo, R., Basta, T., Rachel, R., et al. (2006b). Structural and genomic properties of the hyperthermophilic archaeal virus ATV with an extracellular stage of the reproductive cycle. J. Mol. Biol. 359, 1203-1216. doi: 10.1016/j.jmb.2006.04.027

Prangishvili, D., and Garrett, R. A. (2005). Viruses of hyperthermophilic Crenarchaea. Trends Microbiol. 13, 535-542. doi: 10.1016/j.tim.2005.08.013

Prangishvili, D., Koonin, E. V., and Krupovic, M. (2013). Genomics and biology of Rudiviruses, a model for the study of virus-host interactions in Archaea. Biochem. Soc. Trans. 41, 443-450. doi: 10.1042/BST20120313
Prangishvili, D., and Krupovic, M. (2012). A new proposed taxon for doublestranded DNA viruses, the order "Ligamenvirales". Arch. Virol. 157, 791-795. doi: $10.1007 / \mathrm{s} 00705-012-1229-7$

Prangishvili, D., and Quax, T. E. (2011). Exceptional virion release mechanism: one more surprise from archaeal viruses. Curr. Opin. Microbiol. 14, 315-320. doi: 10.1016/j.mib.2011.04.006

Quax, T. E., Krupovic, M., Lucas, S., Forterre, P., and Prangishvili, D. (2010). The Sulfolobus rod-shaped virus 2 encodes a prominent structural component of the unique virion release system in Archaea. Virology 404, 1-4. doi: 10.1016/j.virol.2010.04.020

Quax, T. E., Lucas, S., Reimann, J., Pehau-Arnaudet, G., Prevost, M. C., Forterre, P., et al. (2011). Simple and elegant design of a virion egress structure in Archaea. Proc. Natl. Acad. Sci. U.S.A. 108, 3354-3359. doi: 10.1073/pnas. 1018052108

Quax, T. E., Voet, M., Sismeiro, O., Dillies, M. A., Jagla, B., Coppee, J. Y., et al. (2013). Massive activation of archaeal defense genes during viral infection. $J$. Virol. 87, 8419-8428. doi: 10.1128/jvi.01020-13

Quemin, E. R., Lucas, S., Daum, B., Quax, T. E., Kuhlbrandt, W., Forterre, P., et al. (2013). First insights into the entry process of hyperthermophilic archaeal viruses. J. Virol. 87, 13379-13385. doi: 10.1128/jvi.02742-13

Quemin, E. R., Prangishvili, D., and Krupovic, M. (2014). Hard out there: understanding archaeal virus biology. Future Virol. 9, 703-706. doi: 10.2217/FVL.14.52

Rakonjac, J., Bennett, N. J., Spagnuolo, J., Gagic, D., and Russel, M. (2011). Filamentous bacteriophage: biology, phage display and nanotechnology applications. Curr. Issues Mol. Biol 13, 51-76.

Rakonjac, J., Feng, J., and Model, P. (1999). Filamentous phage are released from the bacterial membrane by a two-step mechanism involving a short Cterminal fragment of pIII. J. Mol. Biol. 289, 1253-1265. doi: 10.1006/jmbi.1999. 2851

Redder, P., Peng, X., Brugger, K., Shah, S. A., Roesch, F., Greve, B., et al. (2009). Four newly isolated fuselloviruses from extreme geothermal environments reveal unusual morphologies and a possible interviral recombination mechanism. Environ. Microbiol. 11, 2849-2862. doi: 10.1111/j.1462-2920.2009. 02009.x

Reddy, B. L., and Saier, M. H. Jr. (2013). Topological and phylogenetic analyses of bacterial holin families and superfamilies. Biochim. Biophys. Acta 1828, 2654-2671. doi: 10.1016/j.bbamem.2013.07.004

Rice, G., Tang, L., Stedman, K., Roberto, F., Spuhler, J., Gillitzer, E., et al. (2004). The structure of a thermophilic archaeal virus shows a double-stranded DNA viral capsid type that spans all domains of life. Proc. Natl. Acad. Sci. U.S.A. 101, 7716-7720. doi: 10.1073/pnas.0401773101

Roine, E., and Bamford, D. H. (2012). Lipids of archaeal viruses. Archaea 2012, 384919. doi: 10.1155/2012/384919

Roine, E., Kukkaro, P., Paulin, L., Laurinavicius, S., Domanska, A., Somerharju, P., et al. (2010). New, closely related haloarchaeal viral elements with different nucleic Acid types. J. Virol. 84, 3682-3689. doi: 10.1128/jvi.01879-09

Rossman, J. S., and Lamb, R. A. (2013). Viral membrane scission. Annu. Rev. Cell Dev. Biol. 29, 551-569. doi: 10.1146/annurev-cellbio-101011155838

Russel, M., and Model, P. (1989). Genetic analysis of the filamentous bacteriophage packaging signal and of the proteins that interact with it. J. Virol. 63, 3284-3295.

Saier, M. H. Jr., and Reddy, B. L. (2015). Holins in bacteria, eukaryotes, and archaea: multifunctional xenologues with potential biotechnological and biomedical applications. J. Bacteriol. 197, 7-17. doi: 10.1128/jb.02046-14

Samson, R. Y., Obita, T., Freund, S. M., Williams, R. L., and Bell, S. D. (2008). A role for the ESCRT system in cell division in archaea. Science 322, 1710-1713. doi: $10.1126 /$ science. 1165322

Schleper, C., Kubo, K., and Zillig, W. (1992). The particle SSV1 from the extremely thermophilic archaeon Sulfolobus is a virus: demonstration of infectivity and of transfection with viral DNA. Proc. Natl. Acad. Sci. U.S.A. 89, 7645-7649. doi: 10.1073/pnas.89.16.7645

Snyder, J. C., Brumfield, S. K., Kerchner, K. M., Quax, T. E., Prangishvili, D., and Young, M. J. (2013a). Insights into a viral lytic pathway from an archaeal virus-host system. J. Virol. 87, 2186-2192. doi: 10.1128/jvi.02956-12

Snyder, J. C., Samson, R. Y., Brumfield, S. K., Bell, S. D., and Young, M. J. (2013b). Functional interplay between a virus and the ESCRT machinery in archaea. Proc. Natl. Acad. Sci. U.S.A. 110, 10783-10787. doi: 10.1073/pnas.1301605110 
Snyder, J. C., Brumfield, S. K., Peng, N., She, Q., and Young, M. J. (2011). Sulfolobus turreted icosahedral virus $\mathrm{c} 92$ protein responsible for the formation of pyramidlike cellular lysis structures. J. Virol. 85, 6287-6292. doi: 10.1128/jvi.00379-11

Soler, N., Marguet, E., Verbavatz, J. M., and Forterre, P. (2008). Viruslike vesicles and extracellular DNA produced by hyperthermophilic archaea of the order Thermococcales. Res. Microbiol. 159, 390-399. doi: 10.1016/j.resmic.2008.04.015

Stedman, K. M., Deyoung, M., Saha, M., Sherman, M. B., and Morais, M. C. (2015). Structural insights into the architecture of the hyperthermophilic Fusellovirus SSV1. Virology 474, 105-109. doi: 10.1016/j.virol.2014.10.014

Suttle, C. A. (2007). Marine viruses-major players in the global ecosystem. Nat. Rev. Microbiol. 5, 801-812. doi: 10.1038/nrmicro1750

Torsvik, T., and Dundas, I. D. (1974). Bacteriophage of Halobacterium salinarium. Nature 248, 680-681. doi: 10.1038/248680a0

Typas, A., Banzhaf, M., Gross, C. A., and Vollmer, W. (2012). From the regulation of peptidoglycan synthesis to bacterial growth and morphology. Nat. Rev. Microbiol. 10, 123-136. doi: 10.1038/nrmicro2677

Vaney, M. C., and Rey, F. A. (2011). Class II enveloped viruses. Cell. Microbiol. 13, 1451-1459. doi: 10.1111/j.1462-5822.2011.01653.x

Veesler, D., Ng, T. S., Sendamarai, A. K., Eilers, B. J., Lawrence, C. M., Lok, S. M., et al. (2013). Atomic structure of the $75 \mathrm{MDa}$ extremophile Sulfolobus turreted icosahedral virus determined by CryoEM and X-ray crystallography. Proc. Natl. Acad. Sci. U.S.A. 110, 5504-5509. doi: 10.1073/pnas.1300601110

Visweswaran, G. R., Dijkstra, B. W., and Kok, J. (2011). Murein and pseudomurein cell wall binding domains of bacteria and archaea-a comparative view. Appl. Microbiol. Biotechnol. 92, 921-928. doi: 10.1007/s00253-011-3637-0
Votteler, J., and Sundquist, W. I. (2013). Virus budding and the ESCRT pathway. Cell Host Microbe 14, 232-241. doi: 10.1016/j.chom.2013.08. 012

Wais, A. C., Kon, M., Macdonald, R. E., and Stollar, B. D. (1975). Salt-dependent bacteriophage infecting Halobacterium cutirubrum and H. halobium. Nature 256, 314-315. doi: 10.1038/256314a0

Weissenhorn, W., Poudevigne, E., Effantin, G., and Bassereau, P. (2013). How to get out: ssRNA enveloped viruses and membrane fission. Curr. Opin. Virol. 3, 159-167. doi: 10.1016/j.coviro.2013.03.011

White, R., Chiba, S., Pang, T., Dewey, J. S., Savva, C. G., Holzenburg, A., et al. (2011). Holin triggering in real time. Proc. Natl. Acad. Sci. U.S.A. 108, 798-803. doi: $10.1073 /$ pnas. 1011921108

Young, R. (2013). Phage lysis: do we have the hole story yet? Curr. Opin. Microbiol. 16, 790-797. doi: 10.1016/j.mib.2013.08.008

Conflict of Interest Statement: The authors declare that the research was conducted in the absence of any commercial or financial relationships that could be construed as a potential conflict of interest.

Copyright $\odot 2015$ Quemin and Quax. This is an open-access article distributed under the terms of the Creative Commons Attribution License (CC BY). The use, distribution or reproduction in other forums is permitted, provided the original author(s) or licensor are credited and that the original publication in this journal is cited, in accordance with accepted academic practice. No use, distribution or reproduction is permitted which does not comply with these terms. 